\title{
Limitation of the Lubricating Ability of Total Hip Prostheses with Hard on Hard Sliding Material
}

\author{
Kensei Tanaka ${ }^{1)}$, Daichi Uchijima ${ }^{1)}$, Kou Hasegawa ${ }^{1)}$, \\ Takehiro Katori ${ }^{2}$, Rina Sakai ${ }^{1,2)}$, and Kiyoshi Mabuchi ${ }^{1,2)^{*}}$ \\ ${ }^{1)}$ Graduate School of Medical Sciences, Kitasato University \\ 1-15-1 Kitasato, Minami-ku, Sagamihara, Kanagawa 252-0373, Japan \\ ${ }^{2}$ School of Allied Health Sciences, Kitasato University \\ 1-15-1 Kitasato, Minami-ku, Sagamihara, Kanagawa 252-0373, Japan \\ *Corresponding author: km@kitasato-u.ac.jp
}

( Manuscript received 9 March 2012; accepted 21 September 2013; published 31 October 2013 )

(Presented at the Technical Session in the International Conference Hiroshima 2011 )

\begin{abstract}
Recently, metal on metal hip prostheses have been revived following excellent clinical results. However, the risk of wear diseases has not been removed yet. Fluid film formation is necessary for long term reliability of a total hip prosthesis because solid contact of the prosthesis induces serious wear. However, the fluid film can be only formed under limited conditions together with an appropriate shape design. Radial clearance is also an important factor because the radius has an upper limit in the joint space. A radial clearance that is too small induces severe contact, whereas decreasing radial clearance enhances squeezed film formation. The most appropriate radial clearance currently remains unclear. We prepared some specimens with high accuracy of polishing, which were balls and cups of the Co-Cr-Mo alloy with a common radius of $16 \mathrm{~mm}$ and several radial clearances, $0.15-95.8 \mu \mathrm{m}$. Their lubricating ability was evaluated by a friction test using a pendulum machine. The frictional coefficient was a local minimum where the radial clearance was 20-30 $\mu \mathrm{m}$. It was found that appropriate radial clearance of a total hip prosthesis with hard on hard material must exist within this range.
\end{abstract}

Keywords: fluid film formation, frictional coefficient, radial clearance

\section{Introduction}

In the 1980s, the use of metal on metal hip prostheses of the first-generation declined because of metal sensitivities $[1,2]$ and complications from wear debris. In the 1990s, they were revived as a second-generation following excellent clinical results over 30 years $[3,4]$. The risk of wear diseases has not yet been removed. Some clinical complications, which must be caused by wear debris [5], were observed around metal on metal hip prostheses.

Before these findings, it was obvious that solid contact occurred in metal on metal sliding surfaces induced larger wear than polyethylene sliding surfaces. Therefore, fluid film formation is essential for lubrication in a joint prosthesis that contains hard on hard sliding surfaces.

Under any mechanism, such as squeeze films or wedge films, lowering the fluid pressure is preferable to supporting the fluid film in a total hip prosthesis. Because joint resultant force cannot be controlled, the only way to lower pressure in a joint prosthesis is to change the shape design. However, the apparent contact area cannot be widened freely by an increase in head diameter because the outer size of a joint prosthesis is limited in joint space. A possible way to lower pressure is improvements in conformity by decreasing radial clearance.

However, decreasing radial clearance requires a high level of profile accuracy. The unexpected solid contact of a ball with a cup must occur if the size of surface irregularities exceeds radial clearance. Therefore, we must choose appropriate radial clearance between a ball and a cup to realize fluid film lubrication after the sliding surfaces are processed as accurately as possible.

In the present study, we assessed the lubricating ability of different balls and cups of the Co-Cr-Mo alloy with common diameters, different radial clearances, and different surface accuracies in order to investigate the relationship between these design parameters by a pendulum-type friction tester. Three types of hip prostheses with balls and cups of the Co-Cr-Mo alloy 
were added as specimens with different design parameters as representatives of prostheses that are actually used now. We discussed whether metal-on-metal can form sliding surfaces with sufficient lubricating ability or not.

\section{Experimental methods}

Simple spherical models are useful when we approach the shape design of sliding surfaces in joint prostheses. We prepared 18 balls and 14 cups of the Co-Cr-Mo alloy with high quality polishing after precision machining (Fig. 1, Table 1). The diameter was commonly $32 \mathrm{~mm}$ with some variations.

Lubricating ability was evaluated by a friction test using a pendulum machine, as shown in Fig. 2. The cup was set in a bath of saline solution, which was set on the top of a base column. The ball was attached to the upper section of the pendulum frame. The pendulum frame was put on, as the ball got into the cup before the

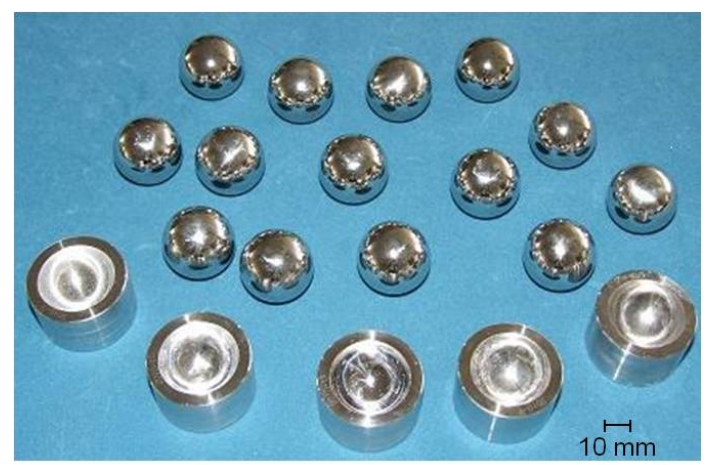

Fig. 1 Balls and cups of Co-Cr-Mo alloy with a common radius of $16 \mathrm{~mm}$ and different radial clearances from 0.15 to $95.8 \mu \mathrm{m}$ measurement started. Vertical force on the sliding surface was applied by the weight on the lower section of the pendulum frame. A biaxial-inclinometer (NS-30/DPG2-RUN, Measurement Specialties, Inc., VA) was set on the top of the pendulum frame and was used for electrical detection of the inclination of the pendulum frame during libration. The voltage signal was digitized in the inclinometer. Data were transferred to a data-acquisition-computer (BX407A4, ONKYO Co., Osaka) on the lower section of the pendulum through a USB-RS232C converter (USB-RSA02, IO DATA Co., Tokyo) with the baud rate of 9600 and 10 times per second.

Measurement started with manual traction of the pendulum frame at an initial inclination of about $0.2 \mathrm{rad}$. After that, free libration followed. Progressive decays in amplitude were acquired in the computer during free libration.

We used the approximation to calculate the absolute inclination $\theta$ [rad].

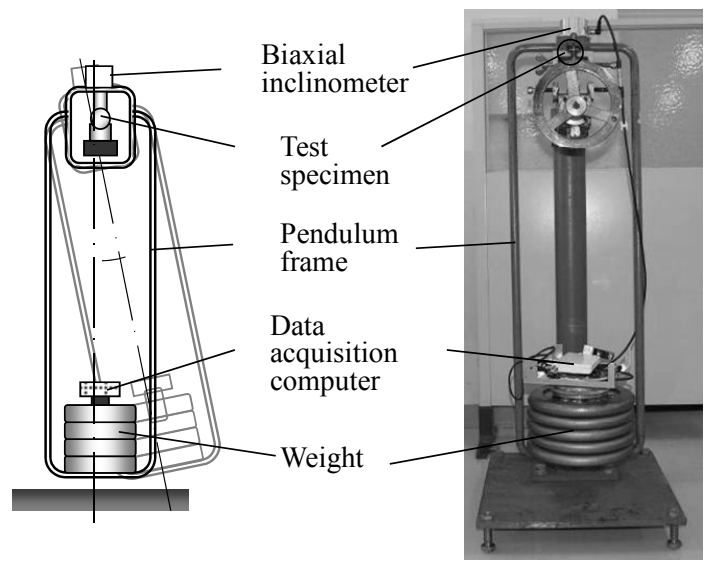

Fig. 2 Pendulum type friction tester

Table 1 Processing accuracy of the specimens. Shape parameters were measured by a coordinate measuring machine (LEGEX574, Mitsutoyo Corp, Kawasaki). Surface roughness, Ra: Center line average, was measured by a digital microscope (VHX-2000, KEYENCE, Osaka)

\begin{tabular}{lllll}
\hline Specimen & Radial clearance $[\mu \mathrm{m}]$ & & Sphericity $[\mu \mathrm{m}]$ & $R a[\mu \mathrm{m}]$ \\
\hline Spherical model & & & & \\
& $0.15-95.8$ & Ball & $0.4-12$ & $0.03-0.05$ \\
& $(171$ patterns $)$ & Cup & $2.6-14.3$ & $0.04-0.07$ \\
A (tri-laminar) & & & & \\
& 47.7 & Ball & 6.3 & 0.0021 \\
& & Cup & 3.7 & 0.0005 \\
B (bi-laminar) & \multirow{4}{*}{41.8} & Ball & 12 & \\
& & Cup & 5.1 & 0.0017 \\
C (bi-laminar) & & & & 0.0006 \\
& 47.5 & Ball & 3 & 0.0021 \\
& & Cup & 10.4 & 0.005 \\
\hline
\end{tabular}




$$
\theta \approx \sqrt{\theta_{x}^{2}+\theta_{y}^{2}}
$$

Here, $\theta_{x}$, and $\theta_{y}$, [rad] are inclinations along the $x$ and $y$ directions, respectively. The error of this approximation was smaller than $1 \%$ because the inclination $\theta$ is under $0.2 \mathrm{rad}$.

From the energy balance between frictional loss and decrease in potential energy of the pendulum, the frictional coefficient $f$ can be calculated as follows.

$$
f=\frac{l\left(\theta_{n}-\theta_{n+1}\right)}{4 r}
$$

Here, $f$ is the frictional coefficient, $\theta_{n}$ is the amplitude at $n$ times libration (Fig. 3), $l[\mathrm{~m}]$ is the distance between the center of gravity and the center of the fulcrum of the pendulum, and $r$ [m] is the radius of the ball. The distant $l$ was $0.806 \mathrm{~m}$ in this pendulum frame.

Period of loading is an important factor in friction tests because squeeze film actions must be dominant mechanisms in lubrication of joint prostheses [6]. Therefore, the first measurement of one series of free libration was performed under a suddenly applied loading condition. Subsequent measurements were continuously performed. Five measurements were performed on each specimen. All measurements were performed at room temperature $\left(23-25^{\circ} \mathrm{C}\right)$.

Additionally, three kinds of commercially available hip prostheses were examined in the same way (Fig. 4, Table 1). One was made of a tri-laminar structure. The other two were made of a bi-laminar structure. None of them were the surface type. Each diameter was $28 \mathrm{~mm}$, $36 \mathrm{~mm}$, or $32 \mathrm{~mm}$.

The design parameter which influenced the frictional coefficient was tested. Polynomial approximation was used in relation to the radial clearance because the relation was not monotonic. Multiple regression analysis was performed on the influence of effective sphericity and effective surface roughness. These factors were defined as integrating irregularities of the two sliding surfaces, respectively. Effective sphericity $S_{E}$ was calculated by the following equation.

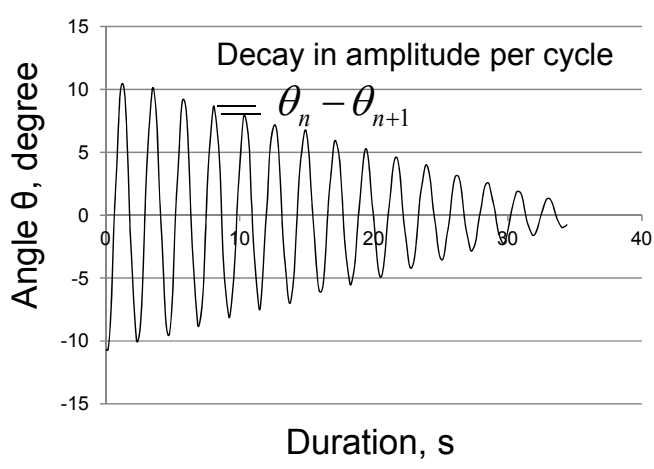

Fig. 3 Detected inclination on the pendulum frame during a free libration

$$
S_{E}=\sqrt{S_{C}^{2}+S_{B}^{2}} \quad[\mu \mathrm{m}]
$$

Here, $S_{C}$ and $S_{B}$ are surface sphericity values of the cup and ball, respectively.

The effective surface roughness $R_{E}$ was calculated by the following equation.

$$
R_{E}=\sqrt{R_{C}^{2}+R_{B}^{2}} \quad[\mu \mathrm{m}]
$$

Here, $R_{C}$ and $R_{B}$ are arithmetic average roughness: $R a$, the center line average, of the cup and ball, respectively.

\section{Results}

Measured frictional coefficients, which were dispersed from 0.122 to 1.05 , did not monotonically relate to the radial clearance. Small radial clearance, which can be good for fluid film formation, did not directly favor low friction. Rather, some results of radial clearances under $10 \mu \mathrm{m}$ exceeded over 1.0, which is larger than usual metal surfaces under wet conditions. On the other hand, small frictional coefficients of about 0.1 were frequently measured in the range of radial clearance from $10 \mu \mathrm{m}$ to $30 \mu \mathrm{m}$. As a whole, friction decreased with increases in radial clearance under $10 \mu \mathrm{m}$ and
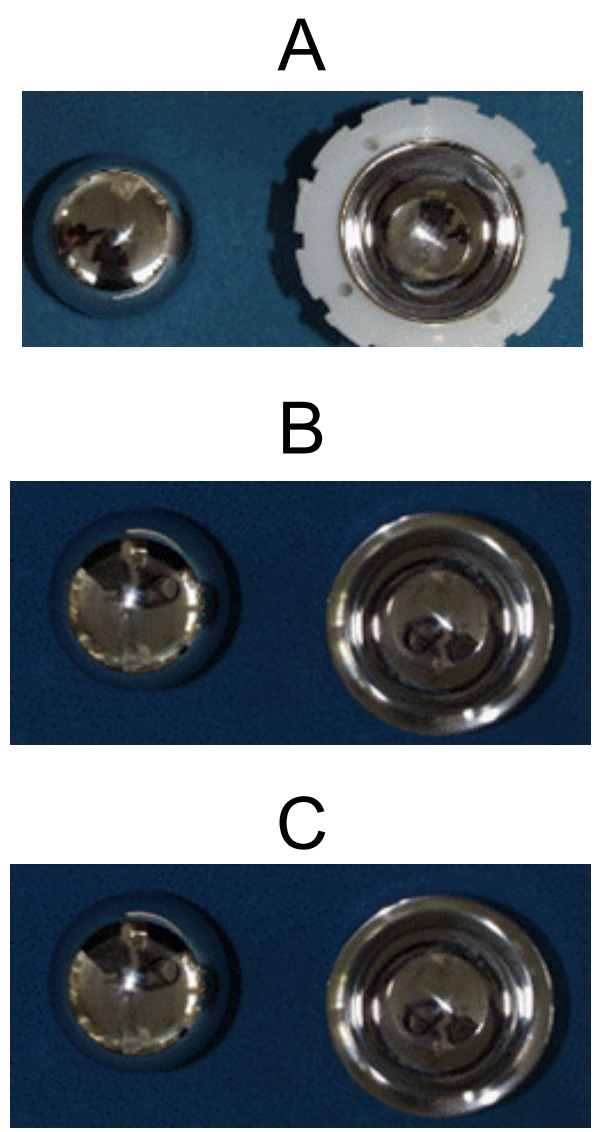

Fig. 4 Three kinds of commercially available hip prostheses 


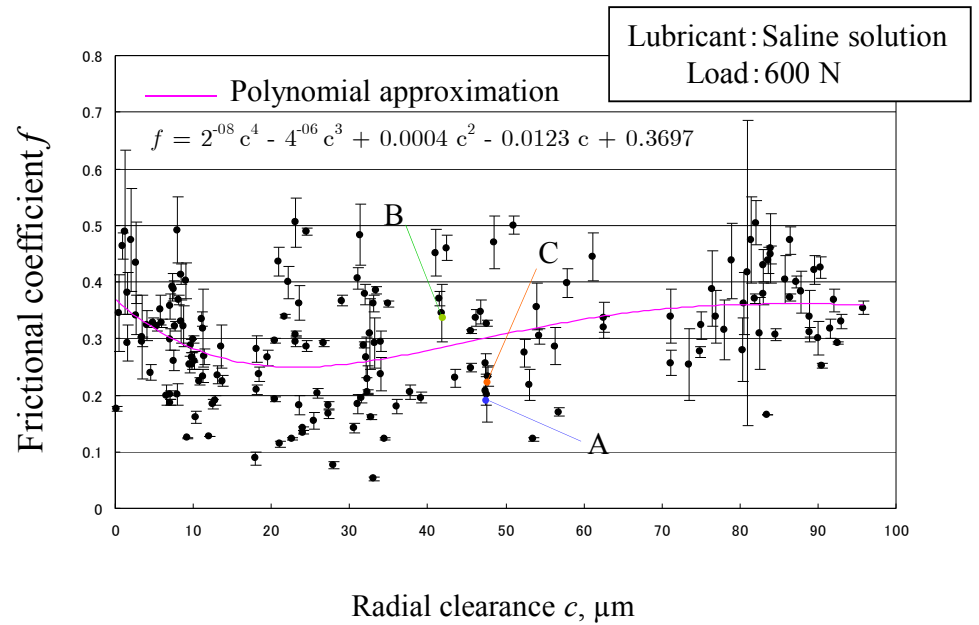

Fig. 5 Frictional coefficients on 171 combinations of 18 balls and 14 cups plotted for each radial clearance. A, B, and $\mathrm{C}$ are the results on commercially available hip prostheses

increased with increases in radical clearance over $30 \mu \mathrm{m}$. Therefore, polynomial approximation was suitable to fit the relationship of friction to radial clearance, as shown in Fig. 5.

Measured frictional coefficients did not show a clear correlation with effective sphericity, as shown in Fig. 6. The multiple regression coefficient was 0.03 . The correlation between frictional coefficient and effective sphericity was not significant.

Measured frictional coefficients showed little positive correlation with effective surface roughness, as shown in Fig. 7. The correlation coefficient was 0.14.

\section{Discussion}

In the present study, we made prototypes of total joint prostheses with high quality to realize fluid film lubrication. The frictional coefficient was used for the

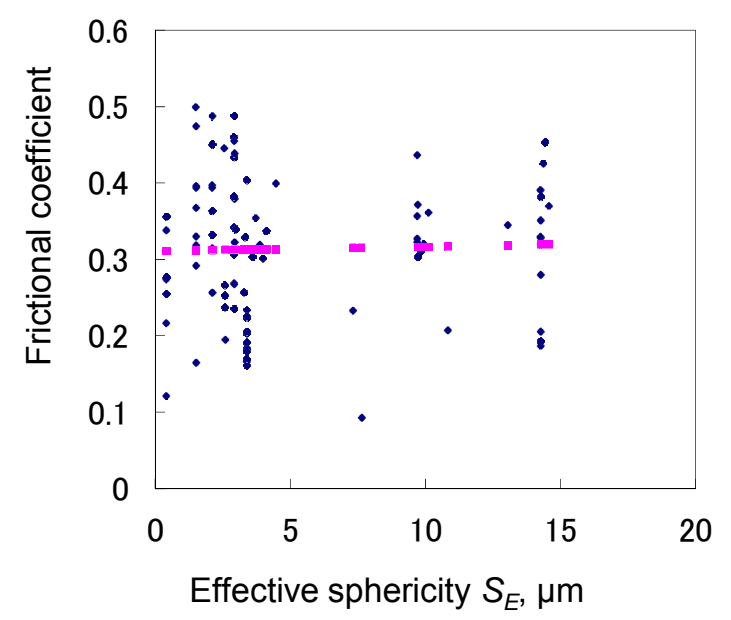

Fig. 6 Results of multiple regression analysis between frictional coefficients and the effective sphericity assessment of lubricating ability. From the results, frictional coefficient values on some prototypes were able to reach smaller than 0.1 . This value was much lower than the values on usual solid sliding surfaces [7]. However, it was higher than well lubricated surfaces like articular cartilage [8]. These results mean that we cannot, at this time, produce a hip prosthesis with sufficient lubricating ability, which can assure long durability in clinical use.

If we aim at further improvements in lubricating ability, assessment of the decisive factors on lubricating ability is necessary. Important design parameters of joint prostheses regarding fluid film lubrication are radius and radial clearance [8]. A large radius is favorable for fluid film formation because it lowers the mean pressure. However, radius size was limited because the joint space was narrow. On the other hand, radial clearance

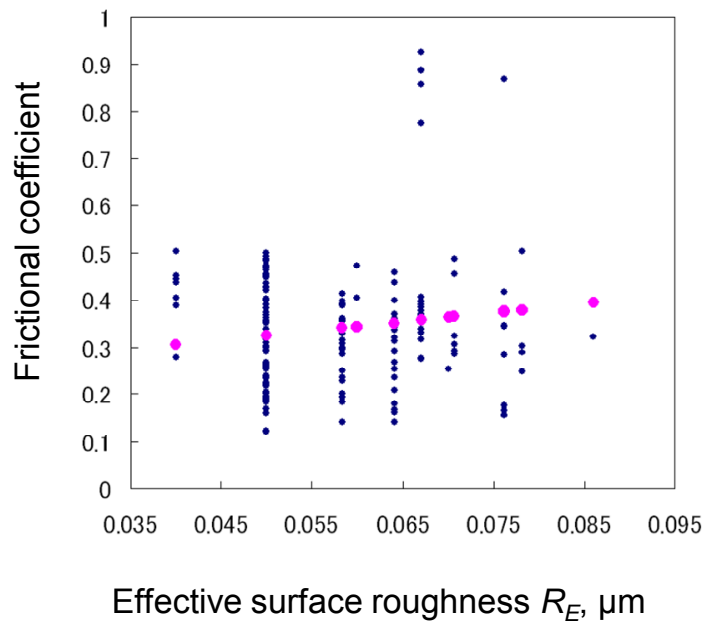

Fig. 7 Results of multiple regression analysis between frictional coefficients and the effective surface roughness 
can be designed to be as small as is needed. Therefore, in the present study, we firstly assessed radial clearance.

Frictional coefficient did not have a simple linear relationship with radial clearance. Polynomial approximation showed that the frictional coefficient becomes a local minimum when radial clearance of a specimen is $20-30 \mu \mathrm{m}$. It was found that appropriate radial clearance of a total hip prosthesis with hard on hard material must exist within this range.

This result disagrees with the simple estimation that the smaller the radial clearance, which makes contact pressure lower, the better the fluid film lubrication ability $[9,10]$. In particular, high friction was measured on some specimens with radial clearance under $5 \mu \mathrm{m}$.

The risk of small radial clearance in a hip prosthesis has been estimated [11]. They reported the clamping phenomenon that induces severe contact at the edge of a cup. The possibility of clamping increases with deformation of a cup. Therefore, the thicker cups become, the lower the risk of cramping due to lower deformation.

If a cup is thick enough, the safety range can be estimated by the Hertzian contact theory. If the Hertzian contact radius exceeds the cup's radius, the fluid film must rupture with the edge contact by cramping.

The Hertzian contact radius $a[\mathrm{~m}]$ at a ball and cup with the same material can be calculated by the following equation $[8,12]$.

$$
\begin{aligned}
& a=\left(\frac{3 W r^{2}}{2 E_{e} c}\right)^{1 / 3} \\
& E_{e}=\left(\frac{E}{1-v^{2}}\right)
\end{aligned}
$$

Here, $W[\mathrm{~N}]$ is the vertical load. $r[\mathrm{~m}]$ is the radius. $E_{e}[\mathrm{~Pa}]$ is the equivalent elastic modulus. $c[\mathrm{~m}]$ is the radial clearance. $E[\mathrm{~Pa}]$ and $v$ are Young's modulus and

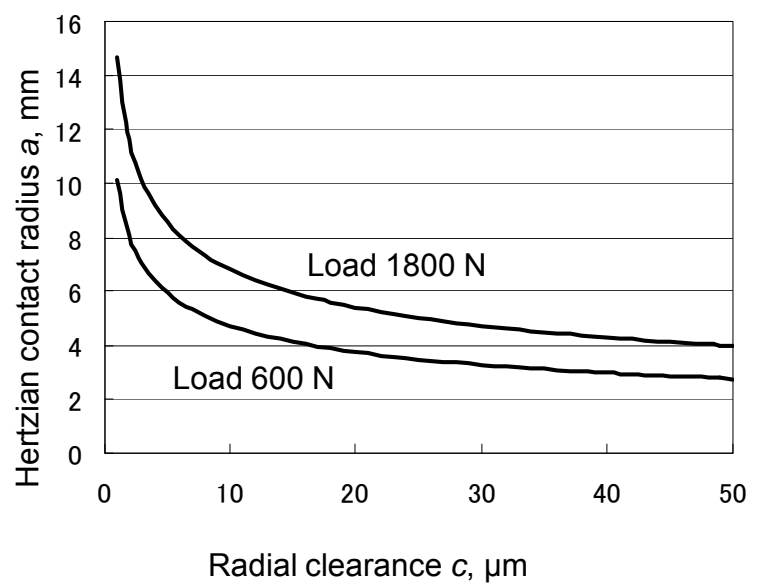

Fig. 8 Hertzian contact radius a calculated at different radial clearances c. The load was $600 \mathrm{~N}$ or $1800 \mathrm{~N}$. The radius was $16 \mathrm{~mm}$. Young's modulus was $230 \mathrm{GPa}$ of Co-Cr-Mo. Poisson's ratio was 0.3
Poisson's ratio of the material, respectively.

The estimated Hertzian contact radius increases drastically when radial clearance was lowered under 10 $\mu \mathrm{m}$ under the conditions of the load $600 \mathrm{~N}$, the radius $16 \mathrm{~mm}$, Young's modulus of the Co-Cr-Mo alloy 230 $\mathrm{GPa}$, and Poisson's ratio $v 0.3$ [13], as shown in Fig. 8. Increases in friction on the specimen with small radial clearance can be explained by this estimation. Furthermore, the load can reach three times the body weight under usual walking conditions [14]. These estimations suggest that the value of radial clearance of $20 \mu \mathrm{m}$, where the minimum frictional coefficient was measured, was critical.

Surface irregularity is another important factor influencing fluid film lubrication. In the present study, the influence of sphericity or surface roughness on lubricating ability was assessed. Some correlations were found between the frictional coefficient and effective surface roughness as shown in Fig. 7. Although the correlation for effective surface roughness is low, the improvement in surface roughness may have effectiveness in improvement of fluid film effect, since the $R a$ values for most of test specimens at the present stage are higher than 0.01 micrometer level. These data showed the possibility of improvements in lubricating ability by improving the surface accuracy of joint prostheses.

The vertical load was set at $600 \mathrm{~N}$ in the present experiment. This value was smaller than the resultant force that was estimated to be three times the body weight at $1800 \mathrm{~N}$ during usual walking conditions [8]. On the other hand, the lubricant was saline solution. The viscosity of saline solution, which is the same as water, can be smaller than secondary synovial fluid, which exists around a joint prosthesis [8]. Therefore, the difference in these two experimental conditions with actual conditions could cancel each other out because the lubricating effect by the fluid film is inversely proportional to the vertical load and proportional to the viscosity of the lubricant [8].

\section{Conclusions}

Appropriate radial clearance of a total hip prosthesis was estimated to exist from 20 to $30 \mu \mathrm{m}$. However, sufficient fluid film lubricating ability cannot be realized in metal on metal hip prostheses if surface accuracy is as high as possible at present. The possibility remains that decreasing surface irregularities in joint prostheses improves lubricating ability.

\section{Acknowledgements}

This study was supported by the Japan Society for the Promotion of Science, Grant-in-Aid for Scientific Research (C) No. 23560170. The authors thank staff in Nara Seiko Inc. for their trial manufacture of specimens. 


\section{References}

[1] Elves, M. W., Wilson, J. N., Scales, J. T. and Kemp, H. B. S., "Incidence of Metal Sensitivity in Patients with Total Joint Replacements," British Med. J., 4 5993, 1975, 376-378.

[2] Benson, M. K. D., Goodwin, P. G. and Brostoff, J., "Metal Sensitivity in Patients with Joint Replacement Arthroplasties," British Med. J., 4 5993, 1975, 374-375.

[3] Sieber, H. P., Rieker, C. B. and Kottig, P., "Analysis of 118 Second Generation Metal-on-Metal Retrieved Hip Implants," J. Bone Joint Surg. Br., 81, 1, 1999, 46-50.

[4] Wimmer, M. A., Loos, J., Nassutt, R., Heitkemper, M. and Fischer, A., "The Acting Wear Mechanisms on Metal-on-Metal Hip Joint Bearings: in vitro Results," Wear, 250, 2001, 129-139.

[5] Campbell, P., Ebramzadeh, E., Nelson, S., Takamura, K., De Smet, K. and Amstutz, H. C., "Histological Features of Pseudotumor-like Tissues From Metal-on-Metal Hips," Clin. Orthop. Rel. Res., 468, 9, 2010, 2321-2327.

[6] Mabuchi, K., Tsukamoto, Y., Obara, T. and Yamaguchi, T., "The Effect of Additive Hyaluronic Acid on Animal Joints with Experimentally Reduced Lubricating Ability," J. Biomed. Maters. Res, 28, 8, 1994, 865-870.
[7] Bowden, F. P. and Tabor, D., "The Friction and Lubrication of Solids," Reprinted, Oxford University Press, New York, 2008, 322-327.

[8] Sasada, T., Tsukamoto, Y. and Mabuchi, K., "Biotribology", Sangyo Tosho, Tokyo, 2000, 59-92 (in Japanese).

[9] Mabuchi, K., Ohta, M., Sakai, R. and Ujihira, M., "Appropriate Radial Clearance of Ceramic-on-Ceramic Total Hip Prostheses to Realize Squeeze-Film Lubrication," Clinical Biomechanics, 19, 4, 2004, 362-369.

[10] Jin, Z. M., Dowson, D. and Fisher, J., "Analysis of Fluid Film Lubrication in Artificial Hip Joint Replacements with Surfaces of High Elastic Modulus,” J. Eng. Med., 211, 3, 1997, 247-256.

[11] Kamali, A., "Hip Joint Tribology," Modern Hip Resurfacing, 2009, 79-89.

[12] Timoshenko, S. P. and Goodier, J. N., "Theory of Elasticity," McGraw-Hill Kogakusha, Tokyo, 1970, 409-420.

[13] Davidson, J. A., Mishra, A. K., Kovacs, P. and Poggie, R. A., "New Surface-Hardened, Low-Modulus, Corrosion-Resistant Ti-13Nb-13Zr Alloy for Total Hip Arthroplasty," Biomed. Mater. Eng., 4, 3, 1994, 231-43.

[14] Mabuchi, K. "Resultant Force on a Hip Joint and Athletic Performance," J. Clinical Sports Medicine, 28, 11, 2011, 1213-1218 (in Japanese). 